\section{Electronic Control}

Cryptography used in Securing Vehicle's Electronic Control Units

\begin{abstract}
Electronic Control Units(ECUs) are know as the brains of a vehicle. A modern vehicle can have over eighty ECUs. All of these "brains" are given a particular task and all of them are controlled by a Super Master ECU which knows what the vehicle is doing and how it is behaving. These ECUs are vulnerable to attacks. Attacks to the brains of a vehicle can result in causalities. Hence the concept of cryptography is adopted into the field of automobiles.
\end{abstract}

\section{Introduction}

- Cars used to be something that was purely mechanical. As time progressed, the creature comforts , safety and quality of cars improved and hence cars became more and more intelligent.

-Features like Autopilot, ABS, Airbags, keyless ignition, Park Assist, Auto headlamps etc was introduced by OEMs to bring in more comfort in a place we spend our time to get to places. - As features increased, the chance of these features being exploited also increased. People hack their ways into Engine Control Module to boost up fuel air ratio, compressions to draw out unsafe power from the engines, stop airbag from deploying, and so on. So the OEMs had to find a way to secure these units from users to ensure safety of us people.

-Keywords: OEM-Original Equipment

Manufacturer, ABS- Anti-Lock Breaking System
Units(ECUs)

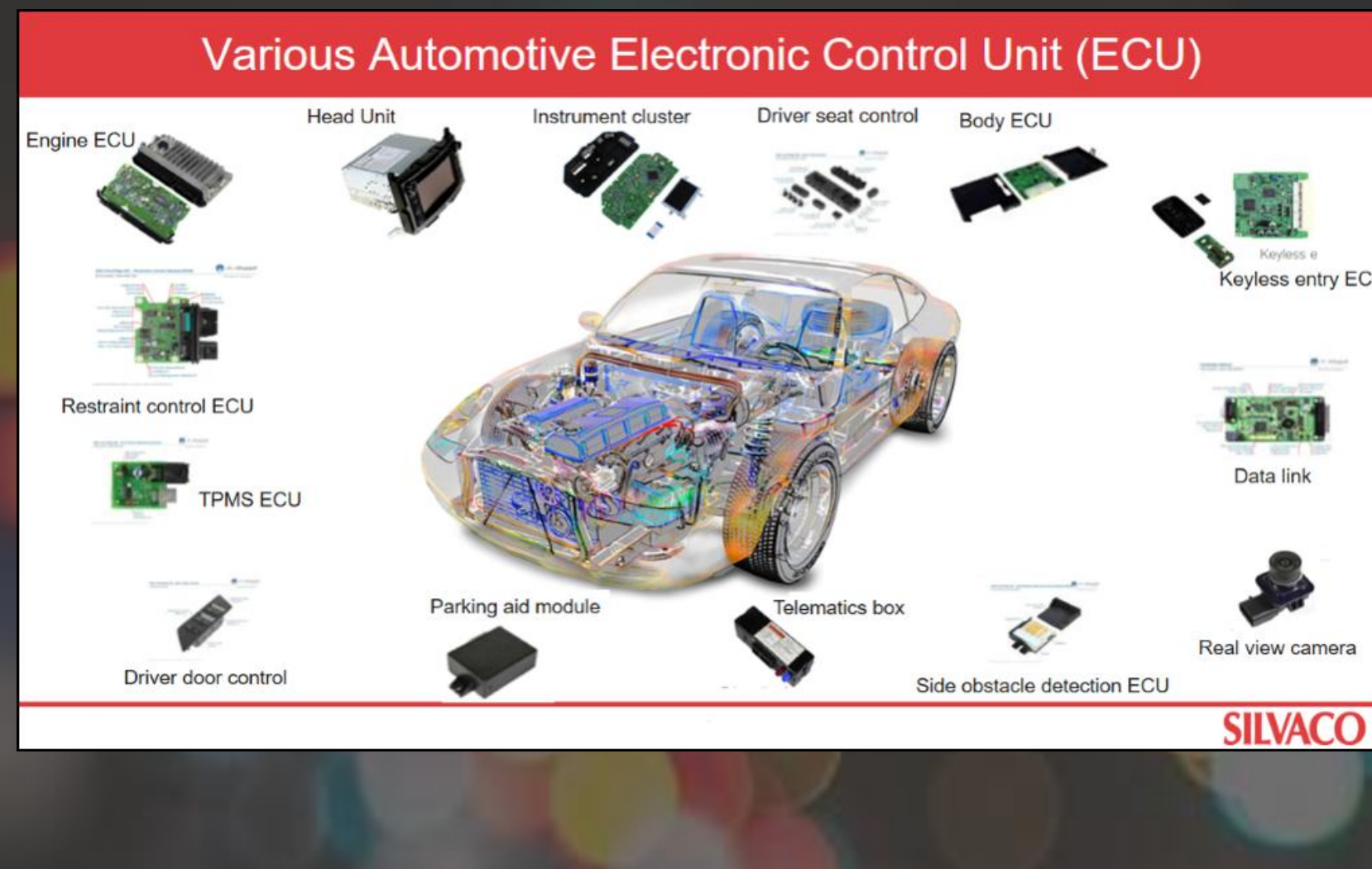

Method for Implementing Security in ECUs

-The key tenets of automotive security are ensuring integrity, authenticity, availability, confidentiality and non-repudiation of the system.

-Integrity and authenticity of the hardware and software in the system, including firmware upgrades and downloaded applications.

-Authenticity, integrity and confidentiality of internal as well as external communications. Confidentiality of stored information.

- Availability of the critical components of the system at all times to ensure functional safety by preventing denial of-service attacks. -Tamper-proof black-box collection of digital forensic data to aid in security breach investigations.

- Security mechanisms to achieve most of the above goals fall broadly into two categories: cryptography-based schemes, and intrusion detection and prevention (IDPS) schemes.

- Secure unlock and Secure programming are the two cyber secure features that can be implemented in an ECU using cryptography based schemes. Secure unlock is necessary to restrict the access to the critical data while secure programming is to make sure that original software code is not modified unless it has secured access to modify the original code
Allen Jacob Siby

Computer Science Engineering Karunya Institute of Technology and Science

-The Electronic Control Units (ECUs) play a critical role in controlling many of the functions of current day's vehicles, as these ECUs are part of the in-vehicle networks, the possibility of security attacks is inevitable.

- To protect the vehicle ECUs against various network attacks, a security architecture based on the notion of master and super master ECUs to ensure ECUs' secure message broadcasting was proposed.

-This architecture was implemented using public key cryptology. The master and super master ECUs also simulated the role of a Key Distribution Center (KDC) through being in charge of generating keys for the units under their control.

- The super master ECU controlled the broadcasting of ECUs' messages from one group of ECUs to the other groups.

-Methods like symmetric key cryptology, Elliptic Curve Cryptology, and stream ciphers were investigated. - Future work will concentrate on the implementation phase. During this phase, the optimal grouping of ECUs will be determined.

-A comparison of the four approaches; public key cryptology, symmetric key cryptology, stream ciphers, and Elliptic Curve cryptology will be carried out to select the most suitable approach for securing the ECUs.

- Furthermore, the most convenient algorithm that takes into consideration the computing resources limitations of the ECUs will be adopted.

Reference:

1. Securing Vehicle's Electronic Control Units by Kevin Daimi 2. Cryptography based cybersecurity implementation in Automotives by Chandrika \& Dr.S.B.Rudraswamy 